\title{
US-Task-Force
}

\section{Jugendliche auf Depressionen screenen!}

\section{Ein Expertengremium aus den USA propagiert das Screening Jugendlicher im Alter zwischen 12 und 18 Jahren auf Depressionen.}

_ Depressionen bei Kindern und Jugendlichen bleiben oft unerkannt. Nur $36 \%$ bis $44 \%$ der Betroffenen erhalten eine adäquate Therapie. Bereits 2009 hat die US Preventive Services Task Force (USPSTF) eine Screening-Empfehlung vorgelegt. Diese wurde jetzt durch neuere Studienergebnisse bestätigt. Demnach ist das Screening bei Jugendlichen im Alter zwischen 12 und 18 Jahren geeignet, eine „Major Depression“ ausreichend zuverlässig zu identifizieren. Den besten Vorhersagewert bescheinigt das Gremium dem PHQ-A (Patient Health Questionnaire for Adolescents). Hierfür werden eine Sensitivität von $73 \%$ und eine Spezifität von $94 \%$ angegeben. Mit dem BDI (Beck-Depressions-Inventar) werden Sensitivitäten von $84 \%-90 \%$ bei Spezifitäten von $81 \%-86 \%$ erreicht.

Zudem besteht laut USPSTF hinreichend Evidenz dafür, dass die aufgrund positiver Screening-Ergebnisse erfolgte Behandlung zu einem "mittelgradigen“ Nutzen führt, d. h. eine Verbesserung der Symptome und des Schweregrads der Depression sowie eine Verbesserung der Funktionalität bewirkt.

Studien guter bzw. ausreichender Qualität liegen dem Gremium zufolge für die Therapie mit selektiven Serotonin-Wiederaufnahme-Hemmern (Fluoxetin bzw. Escitalopram) vor. Eine kognitive Verhaltenstherapie allein zeigte bei Jugendlichen keine deutliche Verbesserung. Dagegen sprach die Kombination aus kognitiver Verhaltenstherapie und Fluoxetin bei $71 \%$ der Teilnehmer an (Ansprechrate Placebo: 35\%). Wie die Experten betonen, habe sich gezeigt, dass das Schadpotenzial einer Pharmakotherapie gering ist, sofern die Patienten sorgfältig beobachtet würden.

Sämtlichen Screening-Studien mit Teilnehmern unter elf Jahren mangelt es an Evidenz. Daher wird das Screening für diese Altersgruppe ausdrücklich nicht empfohlen.

Dr. Elke Oberhofer

- Siu AL et al. Pediatrics 2016;137 (3):e20154467;

doi: 10.1542/peds.2015-4467

\section{Hier steht eine Anzeige.}

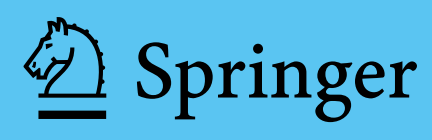

